\title{
Optical Modulation Spectroscopy of Hydrogenated Microcrystalline Silicon
}

\author{
M.F. Cerqueira*, J. Jansen**, G.J. Adriaenssens** and J.A. Ferreira*, \\ * Dep ${ }^{\text {to }}$ de Física, Universidade do Minho-Portugal \\ **Laboratorium Voor Halfgeleiderfysica K.U.Leuven- Belgium
}

\begin{abstract}
The properties of microcrystalline silicon thin films prepared by RF sputtering were investigated by optical modulation spectroscopy at room temperature and the results were correlated with Raman and conductivity measurements. For comparative purposes, a number of good quality PECVD $\mu \mathrm{c}$-Si:H samples were also investigated.

For PECVD samples the OMS signal is very weak, and only measurable for probe beam energies comparable to the gap of amorphous silicon. This indicates the absence of gap states and therefore a very high crystalline fraction, as confirmed by Raman and TEM measurements.

In what concerns RF-sputtered samples, different behaviors can be distinguished: Some samples show a low OMS signal which can be attributed either to a high crystallinity (low density of gap states) or to high recombination rates. These two cases can be distinguished by electrical conductivity values and analysis of the Raman spectra. Other samples exhibit a OMS signal similar to a-Si:H and have low conductivity values, consistent with a Raman spectrum typical of a-Si:H.
\end{abstract}

\section{INTRODUCTION}

One major advantage of microcrystalline silicon over its amorphous family member is its much higher conductivity. Recently industry has focused its interest on microcrystalline silicon, since this advantage can not be neglected. This makes it a promising material to use in solar cells, thin film transistors and light emitting diodes. However, from a theroretical point of view several questions remain unsolved |1|. It is important to know the structure and how it correlates with the optical and the electronic properties of the material. Through the technique of optical modulation 
spectroscopy (OMS) we try to shed some light on which electronic process are involved in opto-electrical properties of these microcrystalline thin films. For the structural characterization we have used Raman spectroscopy, and for the electronic properties steady state conductivity measurements were carried out.

\section{CHARACTERIZATION OF THE SAMPLES}

The sputtered samples were grown on glass substrates with different $\mathrm{Ar}-\mathrm{H}_{2}$ flow rates and $\mathrm{RF}$ sputter power $\mathrm{P}(\mathrm{W})$ therefore having different grain sizes and crystalline fractions. The growth parameters can be seen in table I and experimental details can be found elsewere $|2|$. We verify that the crystalline fraction decreases with increasing RF power and Ar flow |2|. Currently the influence of the $\mathrm{H}_{2}$ flow on the sample quality has not been investigated.

The thickness (d) and refractive index (n) (see table I) of these films were determined through optical transmission and reflection measurements. The results were analyzed according to procedures proposed by Minkov |3| and Swanepoel |4|. Both methods gave comparable results within a $5 \%$ error margin.

TABLE I: Growth conditions, optical parameters (n,d), crystalline size (S), crystalline fraction $(\mathrm{C})$ and conductivities $(\sigma)$ for the sputtered samples

\begin{tabular}{|c|c|c|c|c|c|c|c|c|c|}
\hline Sample & $\begin{array}{c}\mathrm{RF} \\
(\mathrm{W})\end{array}$ & $\begin{array}{c}\mathrm{T} \\
\left({ }^{\circ} \mathrm{C}\right)\end{array}$ & $\begin{array}{c}\mathrm{pH} 2 \\
(\mathrm{~Pa})\end{array}$ & $\begin{array}{c}\mathrm{pAr} \\
(\mathrm{Pa})\end{array}$ & $\begin{array}{c}\mathrm{d} \\
(\mathrm{nm})\end{array}$ & $\mathrm{n}(900 \mathrm{~nm})$ & $\begin{array}{c}\mathrm{S} \\
(\AA)\end{array}$ & $\begin{array}{c}\mathrm{C} \\
(\%)\end{array}$ & $\begin{array}{c}\sigma \\
(\Omega \mathrm{cm})^{-1}\end{array}$ \\
\hline $\mathrm{e} 10$ & 300 & 300 & .15 & 2 & 410 & 2.88 & & & $2.9 \mathrm{E}-7$ \\
\hline $\mathrm{e} 16$ & 300 & 250 & .15 & 2 & 637 & 2.95 & & & $8.7 \mathrm{E}-7$ \\
\hline $\mathrm{e} 13$ & 300 & 250 & .07 & .7 & 524 & 3.39 & & & $9.0 \mathrm{E}-6$ \\
\hline $\mathrm{e} 11$ & 300 & 200 & .15 & 2 & 518 & 2.44 & & & $6.2 \mathrm{E}-7$ \\
\hline $\mathrm{e} 19$ & 180 & 250 & .27 & 5 & 324 & 2.81 & 55 & 61 & $3.8 \mathrm{E}-4$ \\
\hline $\mathrm{e} 4$ & 100 & 250 & .15 & .33 & 200 & 3.05 & 59 & 41 & $8.4 \mathrm{E}-4$ \\
\hline $\mathrm{A} 17$ & 80 & 220 & .39 & .23 & 241 & 2.11 & 60 & 58 & $1.7 \mathrm{E}-2$ \\
\hline
\end{tabular}

Raman scattering was used to provide information about the crystalline fraction and the grain size. The description of Raman setup has been presented elsewhere |2|. The Stokes peak in the vicinity of $520 \mathrm{~cm}^{-1}$ is generally attributed to the transverse optical mode (TO) of crystalline silicon. This peak shifts to smaller wavenumbers for decreasing grain diameter and its position is directly related to the grain size (if we neglect the influence of the stress due to different lattice constants). To analyse the Raman spectra, we used a computer simulation, considering the spectra profile as a 
superposition of the amorphous spectrum and the crystalline spectrum. The crystalline profile was calculated based on the strong phonon confinement $|5,6|$ and a Lorentzian profile was taken for the amorphous TO structure (see figure 1).

To determine the crystalline volume fraction (C) we use

$$
C=\frac{I_{c}}{\left(I_{c}+I_{a}(0.1+\exp (-S / 25 n m))\right.}
$$

where $I_{c}$ is the integrated intensity of the crystalline component and $I_{a}$ is the integrated intensity of the amorphous peak. The average size of the crystallites (S) has to be taken into account as well since it can influence the value for the scattering cross section $|5|$.

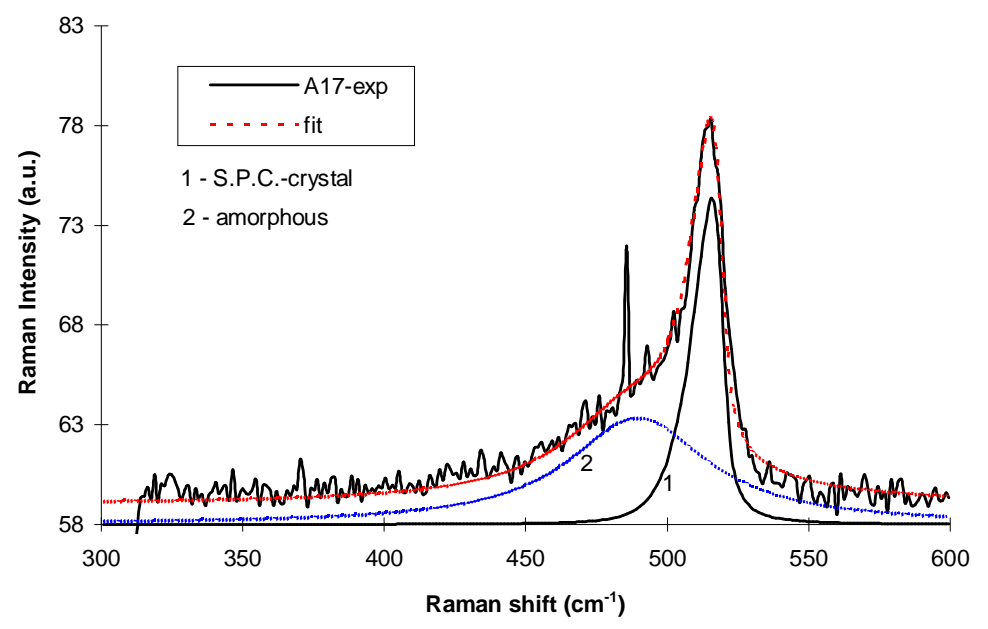

Figure 1 - Deconvolution of the Raman peak of a typical $\mu \mathrm{c}-\mathrm{SI}: H$ sample

\section{OPTICAL MODULATION SPECTROSCOPY}

Optical modulation spectroscopy (OMS) is a powerful technique used for investigation of gap states in amorphous material. The main idea behind the experiment is that due to reorganization of electrons in the gap the transmission for sub band gap light changes. We have used this technique with the main purpose of studying the influence of the crystallites in the amorphous phase of microcrystalline silicon $(\mu \mathrm{c}-$ Si:H).

The OMS experiment is a dual beam experiment. As a basic setup |6|, a laser is used to excite the sample. Due to this excitation, the overall electron occupation of gap states is changed from a standard electron Fermi distribution to a quasi-Fermi 
distribution. The excitation source is modulated. A second beam (probe) is used to measure the difference in transmission $(\Delta \mathrm{T})$ between the laser irradiated and non irradiated sample by a simple lock-in technique. The difference in absorption (OMS signal) can be calculated as the fraction $\Delta \alpha \propto-\Delta \mathrm{T} / \mathrm{T}|7|$. The probe beam is generally a tungsten halogen lamp followed by a monochromator. $\mathrm{Si}$-, $\mathrm{PbS}$ - or $\mathrm{Ge}$ - detectors are used depending on the wavelength of the probe beam.

The difference in absorption can be related to the difference in occupation of electron states between an excited and a non-excited state. From theory we get $|8|$

$$
\Delta \alpha(\hbar \omega) \propto \int g(E) g(E+\hbar \omega)[\Delta f(E)-\Delta f(E+\hbar \omega)] \mathrm{dE}
$$

where $\Delta f(E)$ and $g(E)$ represent respectively the electron redistribution and the density of states at energy E. This means that the OMS signal can be influenced by two major factors, firstly the number of states in the gap and secondly how far the quasi-Fermi levels can be shifted in the gap. Although this method allows us to estimate the density of states if a good model for the electron redistribution can be found, in this work we report qualitative results as a function of the crystal size and the crystalline fraction of the samples.

\section{OMS ON MICROCRYSTALLINE MATERIAL}

Microcrystalline material, in contrast to amorphous material, cannot be described as a single homogeneous phase, but rather as a mixture of variable volume fractions of a crystalline phase, an amorphous phase and grain boundaries (interface region between the amorphous and crystalline phases). The volume fraction occupied by the boundary phase depends on the number and also on the size of the crystallites. The fractions of these three phases depend on the growth conditions.

Although the volume fraction of the grain boundaries might form only a small part of the material, their influence on the electronic and opto-electronic characteristics is considerable. The grain boundary phase will contain a large number of recombination centers (due to dangling bonds, lattice deformations and voids) that will influence the conductivity. If electrons are captured in these traps, the interface layer forms a potential barrier separating the amorphous matrix and the crystallites. 
Perfect crystalline material does not show any OMS signal. This is due to the lack of defects, hence the electron redistibution in the electron gap states has to be zero $\Delta \mathrm{f}(\mathrm{E})=0 \Rightarrow \Delta \alpha=0$. Assuming that the grain boundaries account for a very small fraction of the material, only the amorphous phase will contribute to the OMS signal. The OMS curve of a-Si:H material always show two components, namely photoinduced absorption -PA- (if $\Delta \alpha$ increases, since by exciting the sample, the absorption increases) and photoinduced bleaching PB (decrease in absorption).

The amorphous matrix of our samples shows an OMS signal comparable to that of a-Si:H. There are however a number of small changes, such as shifts in energy position of the increase of the OMS signal due to PA and a decrease in signal due to PB. The differences in the experimental results between a-Si:H samples and the amorphous phase of $\mu \mathrm{c}-\mathrm{Si}: \mathrm{H}$ are probably a consequence of the influence on the amorphous phase by the crystallites. For instance because of the stress in the material the energy positions of the defects in the gap will change, inducing a shift in the onset of the PA and PB.

To measure the difference in transmission $\Delta \mathrm{T}$, the laser beam, produced by an $\mathrm{Ar}^{+}$-ion laser with an intensity of around $100 \mathrm{~mW} / \mathrm{cm}^{2}$ is mechanically chopped at a frequency of about $71 \mathrm{~Hz}$. The transmission $\mathrm{T}$ is measured on the same setup using exactly the same optical path. The energy range for the probe beam is between $0.5 \mathrm{eV}$ and $2.1 \mathrm{eV}$.

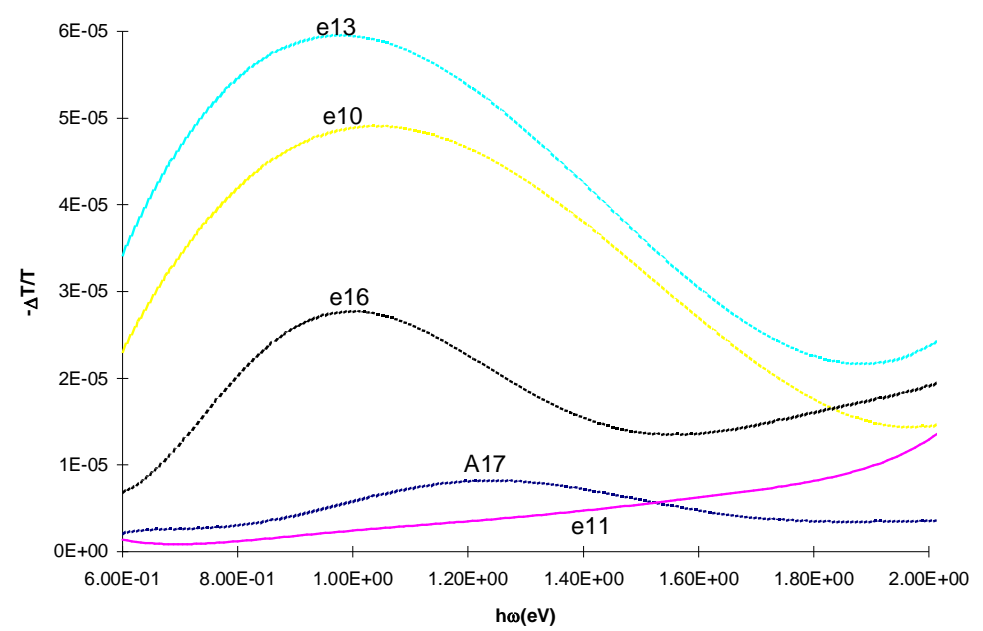


Figure 2 - Different OMS spectra for a number of sputtered $\mu \mathrm{c}-\mathrm{Si}: \mathrm{H}$ samples

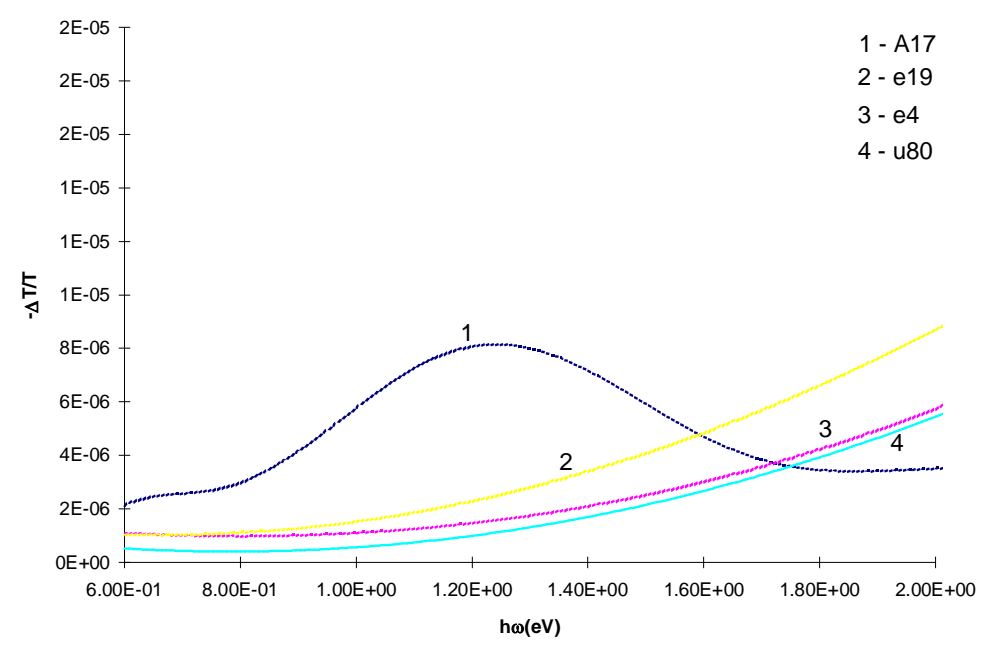

Figure 3 - Comparison of the OMS spectra for good quality sputtered $\mu c-S i: H$ and PECVD prepared $\mu \mathrm{c}-\mathrm{Si}: \mathrm{H}$ samples (u80)

The OMS study of our samples show two kinds of $-\Delta \mathrm{T} / \mathrm{T}$ curves as can be seen in figures 2 and 3. Some samples exhibit a signal with a maximum around $1 \mathrm{eV}$ (typical of a-Si:H). Others show a very low signal only measurable when the probe beam reaches energies comparable with the gap of amorphous silicon. This last kind of behavior was also seen in PECVD samples. If we combine the Raman and conductivity results (see table I), our OMS results can be separated as follows:

I) - OMS curve of small intensity and high conductivity. The crystalline fraction is also high and the grain size is larger than $50 \AA$. If only the amorphous phase contributes, this is exactly what could be expected: the signal is small because the amorphous phase is also small (samples $e 19, e 4$ and A17).

II) - OMS curve of low intensity and low conductivity (sample e11). From Raman measurements in macro geometry this sample is essentially amorphous. Since the number of gap states increases drastically, the recombination through these states will increase proportionally, making electron redistribution difficult. Then we attribute the low OMS signal to the bad quality of the amorphous material, which will result in an enhancement of the recombination rate through the defects in the gap. 
III)- OMS curve with a maximum around $1 \mathrm{eV}$ (equivalent to a-Si:H), showing the presence of the photoinduced absorption $(\Delta \alpha>0)$ and photoinduced bleaching $(\Delta \alpha<0)$ components. The conductivity of these samples $(e 10, e 13, e 16)$ is intermediate between the conductivity of a-Si:H and good quality $\mu \mathrm{c}-\mathrm{Si}: \mathrm{H}$ samples. Althoug the general appearence of the Raman spectra is typical of the amorphous material, a closer investigation by micro-Raman shows the presence of a amount of crystals smaller than $30 \AA$. In view of our assumptions, this strong OMS signal is due to a good quality amorphous phase, which implies a low number of recombination centers $|9|$, although the non negligible presence of the nanocrystals is responsible for the conductivity.

As stated before, small OMS signal could thus mean either a high recombination rate (case II)) or a low number of electron gap states (case I)).

\section{CONCLUDING REMARKS}

In general it is difficult to make a direct correlation between OMS results and the size of the crystals or the crystal fraction. However, if these results are combined with conductivity measurements, some conclusions concerning recombination processes and crystalline fraction can be obtained.

If the crystal fraction is high and consists of large crystallites, the resulting OMS signal is small. This is because the crystalline phase does not influence the OMS signal. Steady state conductivity measurements show conductivity values of the order of $10^{-2}$ to $10^{-4} \mathrm{~S} / \mathrm{cm}$ (e.g. samples $e 4, e 19, A 17$ ). In general we can say that if the crystal phase decreases in size, the OMS signal will increase due to the increase of the amorphous phase in the material. This effect however is cancelled, by the enhancement of recombination in the interface layer between amorphous and crystalline phase, or due to the poor quality of the amorphous phase. The former effect is specially important if the crystalline phase is formed by very small crystals imbedded in the amorphous matrix.

\section{ACKNOWLEDGMENT}

The authors would like to thank F. Finger and R. Carius from ISI-PV Julich for the PECVD samples. M. F. Cerqueira acknowledges a grant from PRAXIS XXI. 


\section{REFERENCES}

|1| - G.J. Adriaenssens, J. Jansen and M. F. Cerqueira, in "Electronic, optoelectronic and magnetic thin films", eds: J. M. Marshall, N. Kirov and A. Vavrek (Research Studies Press LTD, Taunton Somerset England 1995) 235-243

|2| - M. F. Cerqueira, M. Andritschky, L. Rebouta, J. A. Ferreira and M. F. Silva, Vacuum 46 (1995) 1385

|3| - D. A. Minkov, J. Phys. D22 (1985) 199

|4| - R. Swanepoel, J. Phys. E16 (1983) 1214

|5| - E. Bustarret, M. A. Hachichz and H. Brunel, Appl. Phys. Lett. 52 (1988) 1675

$|6|$ - W. Grevendonk, M.Verluyten, J. Dauwen, G.J. Adriaenssens and J. Bezemer, Philos. Mag. B 61 (1990) 393

|7| - P. O’Connor and J. Tauc, Phys. Rev. B25 (1982) 2718

$|8|$ - H. Herremans, J. Jansen and W. Grevendonk, J. Non-Cryst. Solids 164-166 (1993) 347

|9| - A. Mireshghi, W. S. Hong, J. Drewery, T. Jing, S. N. Kaplan, H. K. Lee and V. Perez-Mendez, Mat. Res. Soc. Symp. Proc. 336 (1994) 377 